\title{
Could Non-Linear Heart Rate Variability Analysis of Short RR Intervals Series Give Clinically Valuable Information in Heart Disease?
}

Mourot $\mathrm{L}^{* 1,2}$

${ }^{1}$ Research unit EA4660, Culture Sport Health Society and Exercise Performance Health Innovation platform, Franche-Comté University, France

${ }^{2}$ Clinical Investigation Centre, INSERM CIT 808, CHRU of Besançon 25030, France

*Corresponding author: Mourot L, UFR SMP, 19 rue Ambroise Paré, Plateforme Exercice Performance Santé innovation (EPSI) - Bâtiment Socrate, Fax: +33 3.63.08.23.48, Tel: +33 3.63.08.23.23, E-mail: laurent. mourot@univ-fcomte.fr

Citation: Mourot L (2014) Could Non-Linear Heart Rate Variability Analysis of Short RR Intervals Series Give Clinically Valuable Information in Heart Disease?. J Clin Exp Res Cardiol 1(1): 104. doi: 10.15744/23946504.1.104

Received Date: August 29, 2014 Accepted Date: September 03, 2014 Published Date: September 26, 2014

\begin{abstract}
New analytic methods based on nonlinear system theory have been developed to characterize the nonlinear features in HR dynamics. It is known from long time series (24h ECG recordings) that patients with chronic heart failure or stable coronary heart disease have altered fractal organization in heartbeat dynamics. During such long-time series, many confounding could limit the assessment of autonomic functions. The aim of this study was to test the hypothesis that non-linear indices obtained from short RR intervals series (256 points) can reveal abnormalities in HR behavior in cardiac disease. In 18 healthy subjects, 42 coronary artery disease and 32 chronic heart failure patients, heart rate variability was characterized during supine rest and active standing by spectral analysis, the short- and long-term fluctuations in the R-R interval, approximate and sample entropy and correlation dimension. These indexes are known to evaluate the sympatho-vagal interaction at the heart level as well as the overall complexity of the time series. During supine rest, a greater unpredictability in the R-R interval time series and a lower correlation dimension were observed in cardiac patients. During active standing, they have reduced sympathovagal interaction and complexity. Postural adaptations were blunted in cardiac patients, particularly in chronic heart failure. Our results confirmed the ability of non-linear heart rate variability analysis of short RR intervals series to characterize cardiac disease. They also confirmed that standardization of the conditions of ECG-recording is necessary.
\end{abstract}

Keywords: Heart rate variability; Complexity; Non-linear dynamics; Coronary heart disease; Heart failure

Abbreviations: ApEn-Approximate entropy; HRV-Heart rate variability; CAD-Coronary heart disease; CHF-Chronic heart failure; DFA-Ddetrended fluctuation analysis; HF, LF-Low and High frequency power; pNN50-Fraction of consecutive RR intervals that differ by more than $50 \mathrm{~ms}$; PP-Pulse pressure; rMSSD-root Mean Square of Successive RR interval differences; SampEn-Sample entropy; SAP, DAP, MAP: Systolic, Diastolic, Mean arterial blood pressure

\section{Introduction}

Morbidity and mortality due to chronic heart failure remain high, despite effective drug therapies, implantable cardioverterdefibrillator or resynchronization therapy. Therefore, and despite considerable efforts, risk stratification in patients with heart failure is of great clinical importance. A great number of different measures have been proposed for risk stratification [1] including analysis of heart rate variability (HRV). HRV has been largely used to study the influence of the autonomic drive on the heart in health and disease [2,3]. HRV has been conventionally analyzed with time- and frequency-domain methods, which allowed obtaining information on the sinus node response to parasympathetic and sympathetic activities. A variety of new techniques based on non-linear theories has been proposed to quantify HRV because they may provide useful prognostic information in various clinical settings and their reproducibility may be better than that of traditional indices [4]. Among them, short-term scaling exponent ( $\alpha 1$ ) of detrended fluctuation analysis (DFA) [5] and indices related to the complexity of HRV such as approximate (ApEn) [6] and sample (SampEn) [7] entropy have been studied. These indices provide useful prognostic information [4] and have been proposed to be sensitive to smaller modulations in HR behavior than linear variability measures [8]. Patients suffering of coronary heart disease (CAD) [9,10] or heart failure (HF) [11] have lower HRV than their sedentary counterparts. Patients with chronic HF or stable CAD also have altered fractal organization in heartbeat dynamics [5,12] ApEn, or SampEn have provided information on the vulnerability to atrial fibrillation [13]. These studies analyzed data from long-time series of RR intervals based on ambulatory 24-hours ECG recording. However, such recordings in daily life are not well controlled, nor are they comparable between subjects, and the corresponding analysis of HRV has limited value as an assessment of autonomic functions due to many confounding factors like posture, food intake, wake-sleep cycle, and other physical and mental activities [14]. 
Well controlled resting or dynamic conditions are used to investigate the physiological background of these measures [15-17], but not to characterize heart rate variability in heart disease. This may be due to the fact that during this type of situations, only a limited number of RR intervals can be obtained and the analysis is usually performed on roughly 5 to 10 min of data or 256 cardiac cycles. Such short RR intervals series are considered insufficient to obtain a reliable estimate for most of the non-linear HRV indices used to characterise heart disease. On the other hand, DFA or ApEn have been used with short-term recordings of HRV data, and the results suggested that they can be applied to short data sets $[5,6,12,18]$. Whether or not non-linear HRV indices calculated from short RR intervals series could provide useful information in heart disease remained to determine.

The aim of this study was to test the hypothesis that non-linear HRV indices obtained from short RR intervals series can give clinically valuable information in heart disease.

\section{Materials and Methods}

\section{Population}

Three groups of male subjects participated in the study. Eighteen were healthy: age (mean \pm SD) $51.3 \pm 1.2$ years, height $175.2 \pm 2.7$ $\mathrm{cm}$ and weight $76.1 \pm 0.9 \mathrm{~kg}$. Their medical history and a medical examination were used to discard subjects with cardiovascular, pulmonary or metabolic diseases. The subjects were normotensive and none was taking any medication. Thirty-two were patients suffering from chronic heart failure (CHF; $53.8 \pm 2.4$ years, $172.5 \pm 5.9 \mathrm{~cm}$ and $77.3 \pm 8.7 \mathrm{~kg}$ ) and 42 were patients suffering from coronary heart disease (CAD; $53.9 \pm 3.7$ years, $172.7 \pm 4.3 \mathrm{~cm}$ and $79.5 \pm 13.1 \mathrm{~kg}$ ). CAD patients had preserved left systolic function (ejection fraction $>45 \%$; resting echography- Sequoia C256 Siemens Echocardiography catheter 3V2c-S) and were evaluated after an acute coronary syndrome with or without ST segment elevation. Among them, 28 received ß-blockers, 24 vasodilators, 39 antiplatelets agents, 42 statins and 7 omega 3 fatty acids. CHF patients had left ventricular systolic dysfunction defined as ejection fraction lower than or equal to 45\%. Among them, 29 received B-blockers, 24 vasodilators, 16 diuretic, 4 anti- Aldosterone, 19 anti-platelets agents, 24 statins, 3 omega 3 fatty acids, 5 digital. Heart failure resulted from ischemic cardiomyopathy ( $\mathrm{n}=21$ ) or idiopathic dilated cardiomyopathy $(n=11)$. A coronary angiography was performed in all patients. Patients were included if their clinical condition had been stable for at least three weeks, and if they had stable ECG sinus rhythm.

The study protocol complied with the 1964 Helsinki declaration for human experimentation and was approved by our institutional ethics committee. The subjects were informed of the organization and details of the study and signed an informed consent form.

\section{Organization of the Study}

The subjects were instructed to fast for at least $3 \mathrm{~h}$ before testing, and were asked to refrain from ingesting beverages containing caffeine and alcohol and not to exercise or smoke during the $24 \mathrm{~h}$ preceding each test. The tests were performed between $9 \mathrm{~h} 00$ and $12 \mathrm{~h} 00$.

After an adaptation period of 20 min supine resting, data were recorded in the supine and in upright active posture for 10 min in each position. During each period, ECG data and automatic measurements of systolic (SAP) and diastolic (DAP) arterial blood pressure were taken (BP-8800, Colin Electronics CO., LTD, Japan). Arterial pulse pressure (PP, mmHg) was calculated from SAP minus DAP, and mean blood pressure (MAP, $\mathrm{mmHg}$ ) was calculated as DAP plus one third of PP.

\section{HRV Analyses}

To study heart rate variability (HRV), the duration of RR-intervals was recorded according to procedures already described [16]. Analyses were performed on a time series of 256 cycles selected between minutes 5 to 10 .

HRV analysis was conducted with the aid of Kubios HRV Analysis Software 2.0 for Windows (The Biomedical Signal and Medical Imaging Analysis Group, Department of Applied Physics, University of Kuopio, Finland). For the time domain, the root mean square of successive RR interval differences (rMSSD) and the fraction of consecutive RR intervals that differ by more than 50 ms (pNN50) were reported. For the frequency domain, the normalized low frequency power - LF (n.u.): 0.04-0.15 Hz -, normalized high frequency power - HF (n.u.): $0.15-0.4 \mathrm{~Hz}-$, and the LF/HF ratio were reported.

For non-linear indices, approximate (ApEn) and sample (SampEn) entropy and the short-term fluctuations in the R-R interval data calculated by detrended fluctuation analysis (DFA) were reported. ApEn is a measure that quantifies the amount of overall regularity or predictability in time-series data. Lower ApEn values indicate a more regular (less complex) signal; higher values indicate more irregularity (greater complexity). In this study, complexity was also calculated using sample entropy (SampEn), which has been previously described in detail $[7,19]$.

The fractal nature of the HR time-series allows for measurement of self-similarity correlations using detrended fluctuation analysis (DFA) [19,20] Based on previous research [5] we utilized the short- term ( $\alpha 1 ; 4$ to 16 beats) scaling exponent to analyze our HR time-series data. 


\section{Statistical analysis}

All data are presented as mean \pm SD. Statistical analyses were performed using SigmaStat ${ }^{\circledR}$ software (SPSS Inc, Chicago, USA). Standard statistical methods were used for the calculation of mean \pm SD. Differences between posture (supine vs. standing) and differences between groups (healthy vs. CHF vs. CAD) were tested by a two-way analysis of variance. When interaction or main effects were noted, multiple comparison procedures (Holm-Sidak method) were performed. Differences between groups (healthy vs. CHF vs. CAD) concerning the relative changes during active standing were tested by a one-way analysis of variance on ranks followed by Dunn's test. For bivariate correlation analysis, Pearson's correlation coefficient was calculated. A p-value $<0.05$ was considered significant.

\section{Results}

\section{Hemodynamic data}

In supine position, $\mathrm{HR}$ was lower in CAD than in $\mathrm{CHF}$ or healthy subjects $(\mathrm{p}<0.05)$. No significant differences between groups were observed regarding SAP, DAP and MAP, but the healthy subjects had significantly higher PP. Standing increases HR in the three groups $(\mathrm{p}<0.05)$. The increase was significantly higher in healthy subjects than in the two other groups, and in CAD compared to CHF. Non significant trends for an increase in SAP, DAP and MAP were observed in healthy subjects whereas non significant trends for a decrease were present in CHF and CAD. SAP and DAP were significantly lower in CHF and CAD than in healthy subjects while standing (Table 1).

\begin{tabular}{|c|c|c|c|c|c|c|c|c|c|c|c|}
\hline \multirow{3}{*}{$\begin{array}{c}\text { Absolute changes } \\
\text { HR (bpm) }\end{array}$} & \multicolumn{3}{|c|}{ Healthy subjects } & \multicolumn{4}{|c|}{ Chronic heart failure } & \multicolumn{4}{|c|}{ Coronary artery disease } \\
\hline & \multirow{2}{*}{$\begin{array}{c}\text { Supine } \\
63.2 \pm 8.8\end{array}$} & \multicolumn{2}{|c|}{ Standing } & \multicolumn{2}{|l|}{ Supine } & \multicolumn{2}{|c|}{ Standing } & \multicolumn{2}{|l|}{ Supine } & \multicolumn{2}{|c|}{ Standing } \\
\hline & & $79.9 \pm 12.0$ & $* * *$ & $61.2 \pm 8.3$ & & $66.1 \pm 9.5$ & ${ }^{* *}, \dagger \dagger \dagger$ & $57.2 \pm 5.5$ & $\dagger \dagger$ & $64.4 \pm 5.9$ & $* * *, \dagger \dagger \dagger$ \\
\hline SAP (mmHg) & $118.5 \pm 14.7$ & $122.2 \pm 13.2$ & & $112.0 \pm 11.4$ & & $106.7 \pm 12.0$ & $\dagger \dagger \dagger$ & $113.5 \pm 9.9$ & & $111.7 \pm 13.0$ & $\dagger \dagger \dagger$ \\
\hline $\mathrm{DAP}(\mathrm{mmHg})$ & $69.0 \pm 10.4$ & $75.8 \pm 10.1$ & & $64.7 \pm 8.0$ & & $63.5 \pm 8.7$ & $\dagger \dagger \dagger$ & $67.3 \pm 7.7$ & & $65.0 \pm 7.4$ & $\dagger \dagger \dagger$ \\
\hline MAP (mmHg) & $76.4 \pm 16.0$ & $82.5 \pm 12.8$ & & $80.4 \pm 8.4$ & & $77.9 \pm 9.2$ & & $82.7 \pm 7.9$ & & $80.6 \pm 8.3$ & \\
\hline $\mathrm{PP}(\mathrm{mmHg})$ & $55.3 \pm 9.9$ & $52.0 \pm 10.9$ & & $47.3 \pm 8.3$ & $\dagger \dagger \dagger$ & $43.2 \pm 8.1$ & $\dagger \dagger$ & $46.2 \pm 6.7$ & ††† & $46.7 \pm 10.4$ & \\
\hline \multicolumn{12}{|l|}{ Relative changes } \\
\hline HR (\%) & \multicolumn{3}{|c|}{$27.4 \pm 18.9$} & \multicolumn{3}{|c|}{$5.3 \pm 6.9$} & $\dagger$ & \multicolumn{3}{|c|}{$12.7 \pm 5.1$} & \\
\hline SAP (\%) & \multicolumn{3}{|c|}{$4.7 \pm 14.0$} & \multicolumn{3}{|c|}{$-4.5 \pm 7.8$} & $\dagger$ & \multicolumn{3}{|c|}{$-1.4 \pm 9.8$} & \\
\hline $\operatorname{DAP}(\%)$ & \multicolumn{3}{|c|}{$12.0 \pm 21.4$} & \multicolumn{3}{|c|}{$-2.2 \pm 7.9$} & $\dagger$ & \multicolumn{3}{|c|}{$-2.6 \pm 11.6$} & \\
\hline MAP (\%) & \multicolumn{3}{|c|}{$13.6 \pm 32.4$} & \multicolumn{3}{|c|}{$-3.3 \pm 6.7$} & $\dagger$ & \multicolumn{3}{|c|}{$-2.2 \pm 8.9$} & \\
\hline PP (\%) & \multicolumn{3}{|c|}{$-4.8 \pm 16.3$} & \multicolumn{3}{|c|}{$-6.7 \pm 16.6$} & & \multicolumn{3}{|c|}{$2.8 \pm 26.7$} & \\
\hline
\end{tabular}

Values are mean $\pm \mathrm{SD}$. $\mathrm{HR}=$ heart rate; $\mathrm{SAP}, \mathrm{DAP}, \mathrm{MAP}=$ systolic, diastolic, mean arterial pressure respectively; $\mathrm{PP}=$ pulse pressure.

$*, * *, * * *=$ significantly different from supine at the $0.05,0.01$ and 0.001 level

$\dagger, \uparrow,+\uparrow \dagger=$ significantly different from healthy subjects at the $0.05,0.01$ and 0.001 level

$\ddagger, \ddagger$,

Table 1: Absolute (up) and relative (down) changes in hemodynamic data during head-up tilt

\section{Time domain analysis and Frequency analysis}

rMSSD and pNN50 were significantly lower in CHF and CAD than in healthy subjects. Standing decreased rMSSD and pNN50 $(\mathrm{p}<0.001)$ in healthy subjects and left it unchanged in CHF and CAD (Figure 1, Table 2).

No significant differences were observed between groups in supine posture, except higher HF (n.u.) values in healthy subjects.

Standing significantly increases LF (n.u.) and LF/HF ratio (from $0.9 \pm 1.1$ to $2.9 \pm 4.3$ ) in healthy subjects and CAD (from $1.2 \pm$ 1.1 to $3.0 \pm 2.7$ ), but not in CHF (from $1.9 \pm 1.6$ to $2.9 \pm 2.7$ ). LF (n.u.) was significantly higher in CHF and CAD in the standing position. The relative LF (n.u.) and LF/HF changes were greater in healthy subjects than in CHF and CAD. HF (n.u.) significantly decreased in healthy subjects and CAD while it remained unchanged in $\mathrm{CHF}$.

\section{Non-linear analysis}

al was significantly lower in healthy subjects than in CHF and CAD in supine posture and increased significantly with standing in healthy subjects and CAD.

As regards ApEn, no significant differences between groups were observed in supine posture. A significant decrease was observed in CHF with standing while it remains unchanged in CAD and healthy subjects. ApEn was significantly higher in CAD than in healthy subjects and CHF in upright posture.

SampEn was significantly higher in CAD than in healthy subjects and CHF in supine posture. It significantly decreased on standing in CAD. During standing, SampEn was higher in CHF than in the two other groups (Figure 1, Table 2). 

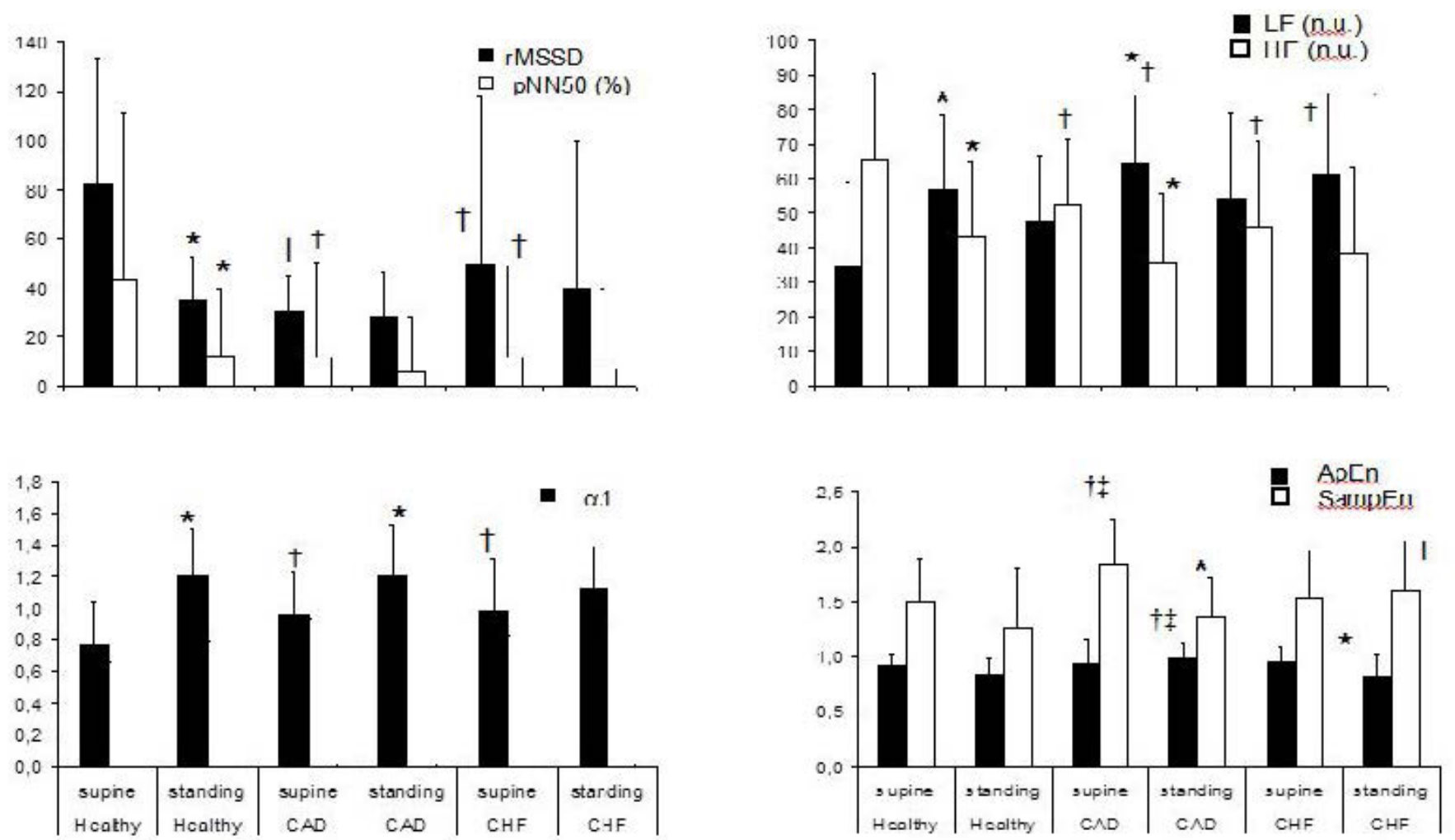

$\mathrm{rMSSD}=$ root mean square of successive RR interval differences; $\mathrm{pNN} 50$ = fraction of consecutive RR intervals that differ by more than $50 \mathrm{~ms} ; \mathrm{LF}=$ low frequency; HF = high frequency; n.u. = normalized units; $\alpha \mathrm{l}=$ short- term fluctuations in the R-R interval; $\mathrm{ApEn}=$ approximate entropy; SampEn =sample entropy. $\mathrm{CAD}=$ coronary artery disease. $\mathrm{CHF}=$ chronic heart failure. * = significantly different from supine

$\dagger=$ significantly different from healthy

\#= significantly different from chronic heart failure

Figure 1: Heart rate variability data in supine and active standing position

\begin{tabular}{|c|c|c|c|c|c|c|c|c|c|c|c|}
\hline \multirow{3}{*}{$\begin{array}{c}\text { Absolute changes } \\
\text { Mean RR (ms) }\end{array}$} & \multicolumn{3}{|c|}{ Healthy subjects } & \multicolumn{4}{|c|}{ Chronic heart failure } & \multicolumn{4}{|c|}{ Coronary artery disease } \\
\hline & \multirow{2}{*}{$\begin{array}{c}\text { Supine } \\
976 \pm 144\end{array}$} & \multicolumn{2}{|c|}{ Standing } & \multicolumn{2}{|l|}{ Supine } & \multicolumn{2}{|c|}{ Standing } & \multicolumn{2}{|c|}{ Supine } & \multicolumn{2}{|c|}{ Standing } \\
\hline & & $773 \pm 129$ & $* * *$ & $1004 \pm 150$ & & $931 \pm 146$ & $* * \dagger \dagger \dagger$ & $1059 \pm 100$ & $\dagger \dagger \dagger$ & $941 \pm 83$ & $* * * \dagger \dagger \dagger$ \\
\hline rMSSD & $82.5 \pm 50.8$ & $35.7 \pm 16.8$ & $* * *$ & $50.0 \pm 68.0$ & $\dagger \dagger \dagger$ & $40.0 \pm 59.7$ & & $30.8 \pm 14.5$ & $\dagger \dagger \dagger$ & $28.1 \pm 18.5$ & \\
\hline pNN50 (\%) & $43.4 \pm 27.9$ & $12.6 \pm 11.0$ & $* * *$ & $11.8 \pm 14.6$ & $\dagger \dagger \dagger$ & $7.2 \pm 12.6$ & & $11.7 \pm 15.2$ & $\dagger \dagger \dagger$ & $5.6 \pm 8.7$ & \\
\hline LF (n.u.) & $34.3 \pm 24.7$ & $56.7 \pm 21.9$ & ** & $54.2 \pm 24.7$ & & $61.4 \pm 24.6$ & $\dagger \dagger$ & $47.4 \pm 19.1$ & & $64.2 \pm 19.9$ & $* * \dagger$ \\
\hline HF (n.u.) & $65.7 \pm 24.7$ & $43.3 \pm 21.9$ & $* *$ & $45.8 \pm 24.7$ & $\dagger$ & $38.6 \pm 24.6$ & & $52.6 \pm 19.1$ & $\dagger \dagger$ & $35.8 \pm 19.9$ & ** \\
\hline $\mathrm{LF} / \mathrm{HF}$ & $0.9 \pm 1.1$ & $2.9 \pm 4.3$ & $* *$ & $1.9 \pm 1.6$ & & $2.9 \pm 2.7$ & & $1.2 \pm 1.1$ & & $3.0 \pm 2.7$ & $* *$ \\
\hline$\alpha 1$ & $0.76 \pm 0.27$ & $1.22 \pm 0.28$ & $* * *$ & $0.98 \pm 0.34$ & $\dagger \dagger \dagger$ & $1.12 \pm 0.37$ & & $0.96 \pm 0.28$ & $\dagger$ & $1.21 \pm 0.32$ & $* * *$ \\
\hline ApEn & $0.92 \pm 0.10$ & $0.84 \pm 0.15$ & & $0.95 \pm 0.14$ & & $0.83 \pm 0.18$ & $* *$ & $0.94 \pm 0.23$ & & $0.98 \pm 0.13$ & ††おおま \\
\hline SampEn & $1.49 \pm 0.40$ & $1.26 \pm 0.54$ & & $1.52 \pm 0.45$ & & $1.60 \pm 0.64$ & $\dagger \dagger$ & $1.84 \pm 0.41$ & $\dagger \dagger \ddagger \ddagger$ & $1.37 \pm 0.35$ & $* * *$ \\
\hline \multicolumn{12}{|l|}{ Relative changes } \\
\hline$\Delta \mathrm{rMSSD}(\%)$ & \multicolumn{2}{|c|}{$39.2 \pm 42.8$} & & \multicolumn{3}{|c|}{$-6,7 \pm 40.4$} & $\dagger$ & \multicolumn{3}{|c|}{$1.3 \pm 74.2$} & $\dagger$ \\
\hline$\Delta \mathrm{pNN} 50(\%)$ & \multicolumn{2}{|c|}{$-33.8 \pm 93.5$} & & \multicolumn{3}{|c|}{$11.2 \pm 132.3$} & & \multicolumn{3}{|c|}{$3.3 \pm 151.7$} & \\
\hline$\Delta \mathrm{LF}(\%)$ & \multicolumn{2}{|c|}{$132.7 \pm 129.1$} & & \multicolumn{3}{|c|}{$35.3 \pm 59.5$} & $\dagger$ & \multicolumn{3}{|c|}{$55.1 \pm 77.5$} & $\dagger$ \\
\hline$\Delta \mathrm{HF}(\%)$ & \multicolumn{2}{|c|}{$-25.5 \pm 50.1$} & & \multicolumn{3}{|c|}{$-15.7 \pm 39.3$} & & \multicolumn{3}{|c|}{$-26.0 \pm 47.3$} & \\
\hline$\Delta \mathrm{LF} / \mathrm{HF}(\%)$ & \multicolumn{2}{|c|}{$652 \pm 1610$} & & \multicolumn{3}{|c|}{$161 \pm 303$} & $\dagger$ & \multicolumn{3}{|c|}{$283 \pm 505$} & $\dagger$ \\
\hline$\Delta \alpha 1(\%)$ & \multicolumn{2}{|c|}{$78.1 \pm 78.9$} & & \multicolumn{3}{|c|}{$33.2 \pm 49.4$} & $\dagger$ & \multicolumn{3}{|c|}{$36.3 \pm 51.3$} & \\
\hline$\triangle \mathrm{ApEn}(\%)$ & \multicolumn{2}{|c|}{$-7.5 \pm 20.6$} & & & $.5 \pm 2$ & & & & $2 \pm 3$ & & $\ddagger$ \\
\hline$\Delta$ SampEn $(\%)$ & -6.3 & & & & $5 \pm 4$ & & & & $2.3 \pm 2$ & & $\ddagger$ \\
\hline
\end{tabular}

$\mathrm{RR}=\mathrm{R}-\mathrm{R}$ intervals; $\mathrm{rMSSD}=$ root mean square of successive RR interval differences; $\mathrm{pNN} 50=$ fraction of consecutive RR intervals that differ by more than $50 \mathrm{~ms} ; \mathrm{LF}=$ low frequency; $\mathrm{HF}=$ high frequency; $\mathrm{n} . \mathrm{u}$. $=$ normalized units; $\alpha \mathrm{l}=$ short-term fluctuations in the R-R interval; ApEn = approximate entropy; SampEn =sample entropy.

$*, * *, * * *=$ significantly different from supine at the $0.05,0.01$ and 0.001 level

$\dagger, \dagger+\uparrow \dagger \dagger=$ significantly different from healthy subjects at the $0.05,0.01$ and 0.001 level

$\$, \ddagger \neq,+\$ \neq=$ significantly different from chronic heart failure at the $0.05,0.01$ and 0.001 level

Table 2: Absolute (up) and relative (down) changes in heart rate variability data during head-up tilt 


\section{Correlations among different HRV indexes}

The correlation coefficients between the different HRV measures in supine and standing postures, and across all conditions (respectively) are shown in Table 3.

\begin{tabular}{|c|c|c|c|c|c|c|c|c|c|c|}
\hline & & Mean RR & RMSSD & pNN50 (\%) & LF (n.u.) & HF (n.u.) & LF/HF & $\alpha 1$ & ApEn & SampEn \\
\hline Supine & Mean RR & 1,00 & $0,337^{\star}$ & $0,364^{*}$ & $-0,09$ & 0,09 & $-0,09$ & $-0,15$ & 0,14 & 0,01 \\
\hline \multirow[t]{8}{*}{$\mathrm{n}=98$} & rMSSD & & 1,00 & $0,922^{*}$ & $-0,51^{*}$ & $0,51^{*}$ & $-0,51^{*}$ & $-0,62^{*}$ & 0,08 & $-0,41^{*}$ \\
\hline & pNN50 (\%) & & & 1,00 & $-0,40^{*}$ & $0,40^{*}$ & $-0,40^{*}$ & $-0,49^{*}$ & 0,12 & $-0,30^{*}$ \\
\hline & LF (n.u.) & & & & 1,00 & $-1,00^{*}$ & $1,00^{*}$ & $0,85^{*}$ & 0,14 & 0,04 \\
\hline & HF (n.u.) & & & & & 1,00 & $-1,00^{\star}$ & $-0,85^{*}$ & $-0,14$ & $-0,04$ \\
\hline & LF/HF & & & & & & 1,00 & $0,85^{*}$ & 0,14 & 0,04 \\
\hline & $\alpha 1$ & & & & & & & 1,00 & 0,07 & 0,06 \\
\hline & ApEn & & & & & & & & 1,00 & $-0,20^{*}$ \\
\hline & SampEn & & & & & & & & & 1,00 \\
\hline Standing & Mean RR & 1,00 & $0,26^{*}$ & $0,20^{*}$ & $-0,07$ & 0,07 & $-0,07$ & $-0,20^{*}$ & $0,27^{\star}$ & $-0,08$ \\
\hline \multirow[t]{8}{*}{$\mathrm{n}=98$} & rMSSD & & 1,00 & $0,88^{\star}$ & $-0,43^{*}$ & $0,43^{*}$ & $-0,43^{\star}$ & $-0,40^{*}$ & 0,00 & $-0,31^{*}$ \\
\hline & pNN50 (\%) & & & 1,00 & $-0,37^{*}$ & $0,37^{*}$ & $-0,37^{\star}$ & $-0,32^{*}$ & 0,05 & $-0,17$ \\
\hline & LF (n.u.) & & & & 1,00 & $-1,00^{*}$ & $1,00^{*}$ & $0,80^{*}$ & 0,12 & $-0,20$ \\
\hline & HF (n.u.) & & & & & 1,00 & $-1,00^{*}$ & $-0,80^{*}$ & $-0,12$ & 0,20 \\
\hline & $\mathrm{LF} / \mathrm{HF}$ & & & & & & 1,00 & $0,80^{*}$ & 0,12 & $-0,20$ \\
\hline & $\alpha 1$ & & & & & & & 1,00 & 0,02 & $-0,22^{*}$ \\
\hline & ApEn & & & & & & & & 1,00 & 0,02 \\
\hline & SampEn & & & & & & & & & 1,00 \\
\hline All & Mean RR & 1,00 & $0,37^{*}$ & $0,37^{*}$ & $-0,20^{*}$ & $0,20^{*}$ & $-0,20^{*}$ & $-0,31^{*}$ & $0,23^{*}$ & 0,06 \\
\hline \multirow[t]{8}{*}{$\mathrm{n}=196$} & rMSSD & & 1,00 & $0,90^{*}$ & $-0,49^{*}$ & $0,49^{*}$ & $-0,49^{*}$ & $-0,55^{*}$ & 0,08 & $-0,28^{*}$ \\
\hline & pNN50 (\%) & & & 1,00 & $-0,41^{*}$ & $0,41^{*}$ & $-0,41^{*}$ & $-0,45^{*}$ & $0,14^{*}$ & $-0,16^{*}$ \\
\hline & LF (n.u.) & & & & 1,00 & $-1,00^{*}$ & $1,00^{*}$ & $0,85^{*}$ & 0,08 & $-0,16^{*}$ \\
\hline & HF (n.u.) & & & & & 1,00 & $-1,00^{*}$ & $-0,85^{*}$ & $-0,08$ & $0,16^{*}$ \\
\hline & $\mathrm{LF} / \mathrm{HF}$ & & & & & & 1,00 & $0,85^{*}$ & 0,08 & $-0,16^{*}$ \\
\hline & $\alpha 1$ & & & & & & & 1,00 & $-0,01$ & $-0,17^{\star}$ \\
\hline & ApEn & & & & & & & & 1,00 & $-0,06$ \\
\hline & SampEn & & & & & & & & & 1,00 \\
\hline
\end{tabular}

Abbreviations, see Table $2 ; \mathrm{n}=$ no. of subjects. ${ }^{*} \mathrm{P}<0.05$.

Table 3: Correlations among all heart rate variability measures during supine and standing conditions, and across all conditions

The scaling exponent al was negatively correlated with HF (n.u.) and positively with LF (n.u.) and LF/HF. The relationships were slightly greater in supine than in standing posture.

\section{Discussion}

Autonomic assessment has played an important role in elucidating the role of the autonomic nervous system in diverse pathological conditions including heart diseases. The anatomic location of the cardiovascular autonomic nervous system renders it inaccessible to simple direct physiological testing. Techniques that are available for the study of autonomic nerve activity in humans do not allow any direct exploration of vagal efferent activity. With regard to sympathetic nerve activity, several studies have indicated the interesting capabilities of two of the most widely used techniques [21] noradrenalin spillover to plasma, and electroneurographic recordings of muscle sympathetic nervous activity. However, neither of these methods appears to be suitable for regular clinical use in large numbers of patients or outside the clinical laboratory. Analysis of spontaneous cardiovascular beat-by-beat variability has gained wide credibility as a means of assessing cardiovascular neural regulation in a variety of physiological and pathophysiological conditions, including myocardial infarction and heart failure $[5,9,12,22]$. A number of studies dealing with HRV have shown that R-R intervals fluctuate in a very complex and apparently erratic manner exhibiting patterns suggestive of non-linear processes [23]. Because of these non-linear components, the R-R interval time series signal cannot be properly assessed using linear techniques such as spectral analysis [23]. In the search for improved methods for decoding hidden information in the R-R interval dynamics, parameters arising from non-linear methods have therefore been identified. These indices provide useful prognostic information [4] but, to our knowledge, have not been used during short-term dynamic conditions designed to evaluate autonomic nervous activity in cardiac disease. We tested the hypothesis that non-linear HRV indices obtained from short RR intervals series can give clinically valuable information in cardiac disease. 
During supine rest, our results were in line with previous reports. A greater unpredictability in the R-R interval time series was observed in cardiac patients [24], which may be related to lower vagal and higher sympathetic RR interval modulations [25]. However, contrary to previous results obtained on a longer data set $[5,8,12]$ we observed a stronger correlation of short term HR dynamics in healthy subjects (i.e., lower $\alpha 1$ of DFA in healthy subjects). Methodological considerations (i.e., length of the data sets) could explain this discrepancy [18] even if it was argues that $\alpha 1$ of DFA could be used with relative short-time series [5,6]. On the other hand, such differences were already reported concerning the effect of age on $\alpha 1$, and were related to differences in the conditions of RR interval recording (position, physical or mental activity, number of RR intervals) [26]. Thus, despite the fact that only $256 \mathrm{RR}$ intervals were used to perform non-linear analysis of HRV, the characterization of RR dynamics in the present study is in line with those previously reported and suggest that ambulatory 24-hours ECG recording is not required to obtained useful prognostic information in heart disease.

The change from supine to erect posture decreases the effective circulating blood volume. One half to one liter of blood contained in the thorax is transferred to the legs and the abdomen, triggering several cardiovascular and autonomic nervous system changes that have been well studied. To summarize, the decrease in central venous pressure, stroke volume and pulse pressure reduces the afferent baroreflex traffic through unloading cardiopulmonary and arterial baroreceptors [16]. Cardiac output, blood pressure and cerebral perfusion are maintained through a decreased cardiac parasympathetic activity and an increased cardiac and vascular sympathetic activity, which increase heart rate and heighten vasoconstriction. The results of this study are entirely in line with these views. In healthy subjects, spectral analysis indicated a shift of the sympathovagal balance toward sympathetic predominance through a sympathetic activation and vagal withdrawal $[16,17,27]$. The pattern of changes was less clear in cardiac disease, with no changes in spectral indexes in CHF. The increase in $\alpha 1$ of DFA in healthy subjects revealed a stronger correlation of short term HR dynamics during standing [16,17]. These increases were not present in CHF. On the other hand, ApEn decreased significantly in CHF and SampEn decreased significantly in CAD, suggesting a decreased overall complexity with standing that was not present in healthy subjects. Complexity indices based on ApEn and SampEn did not change significantly in healthy subjects [17].

Taken together, these results suggest reduced sympathovagal interaction and complexity during active standing in heart disease. Postural adaptations were blunted in cardiac patients, particularly in chronic heart failure, which is likely due to high level of circulating norepinephrine in these patients [28]. Most changes in spectral and DFA indexes were not observed in cardiac disease, showing reduced interaction and competition among subjects' subsystems. Hence, both resting condition and dynamic stimulation allowed distinguishing cardiac patients from healthy subjects.

It has been reported that HRV indexes are highly correlated [17]. Indeed, $\alpha 1$ was related to both weighted and conventional spectral ratios such as $\mathrm{LF} /(\mathrm{HF}+\mathrm{LF})$ and it was claimed that a 1 can no longer be considered to be in a different class of measurement from spectral analysis [29]. However, we showed that taking into account both spectral analysis of HRV and DFA is necessary to describe cardiac autonomic status accurately [16]. The information given by these indexes is not entirely interchangeable. On the other hand, the significant decrease in ApEn and SampEn with active standing in patients was not present in healthy subjects. Together with the fact that ApEn and SampEn had only weak correlations with all HRV measures, the view that an overall complexity measure describes different features in HR dynamics from linear analysis is reinforced. This also suggests that a short-term controlled dynamics challenge could reveal altered heart rate autonomic control in cardiac disease. Another result is that differences observed in the supine resting condition between groups were not always present in the standing posture. This implies that standardization of posture is required during HRV analysis in order to highlight differences in cardiac autonomic function between healthy subjects and those suffering from heart disease. This is strengthened by the fact that fractal and complexity measures of HRV were found to be weakly correlated with the traditional time- and frequency-domain indices when measured from 24-h ambulatory ECG recordings [30], whereas the relationships were stronger during short-term RR recording [17]. This may be due to the uncontrolled condition, which may have significant effects on the characteristics of short-term HR dynamics [17].

The physiological background of the non-linear measures used in the present study is still not well defined. The activity of the heart is under the control of the autonomic nervous system (sympathetic and parasympathetic). The interaction between the two branches is classically characterized as opposite and reciprocal, even if under physiological and pathophysiological circumstances they can be both synchronous and synergistic [16]. Physical or pharmacological interventions such as facial or hand cold water immersion or NE infusion suggest a direct relationship between sympathetic outflow to the heart and a1 [20,31,32]. Also, ApEn and SampEn decrease during sympathetic activation paralleled by vagal withdrawal [27]. On the other hand, no correlation between cardiac norepinephrine spillover and these indices was observed [15], suggesting that they are not reflective of sympathetic neural outflow to the heart. Vagal blockade alters the fractal-like HR dynamics ( $a 1)$ and reduces the complexity (ApEn) of HR behaviour. Therefore, if a1, ApEn or SampEn could be considered an indicator of the sympatho-vagal interaction to the heart, it seems that they are mainly related to the vagal control of heart rate [15,33]. This is in line with the results of study of Baumert et al. who reported that the predictive value of HRV for cardiac risk stratification might lie predominantly in its ability to quantify vagal outflow to the sinus node [15]. 


\section{Limits}

In the present study, respiration was not recorded during the active standing test. This is of concern, especially because both the fractal and complexity measures of HR variability can be significantly influenced by changes in the breathing pattern [33]. The subjects spontaneously adapted their tidal volume and breathing frequency but they were surveyed by one investigator to ensure that the ventilation pattern remained quite steady during all phases of the protocol. As reported earlier [26], we used a posteriori visual inspection of the power spectra to verify the location of the respiratory component in the HF region.

The absence of concomitant short- and long-term R-R recoding in the same subjects, the potential impact of cardiovascular drug [34] as well as the limited number of subjects limits also the interpretation of our results and further researches on the usefulness of short-term dynamic conditions in the diagnosis of cardiac disease are required.

Finally, it should highlight that in this cross-sectional study the prediction of cardiac disease from HRV indices was not addressed and that only an association between changes in HRV indices and cardiac disease was observed. These preliminary results should be confirmed by appropriate longitudinal studies.

\section{Conclusion}

The main result of the present study was that non-linear HRV indices obtained from short RR intervals series (256 points) can give clinically valuable information in cardiac disease to highlight the deficiency of the neurocardiac regulation. Moreover, these indices also revealed that the autonomic nervous system adjustments to postural changes were blunted in cardiac patients, with reduced sympatho-vagal interaction during active standing. Most changes in spectral and DFA indexes were not present in patients. Also, active standing decreased the overall complexity of HRV in cardiac patients.

\section{Acknowledgment}

The authors wish to thank the subjects for their time and cooperation. We would also like to thank Frances Sheppard of the Clinical Investigation Center of Besançon for her linguistic assistance.

\section{References}

1. Bailey SJ, Winyard P, Vanhatalo A, Blackwell JR, Dimenna FJ, et al. (2009) Dietary nitrate supplementation reduces the O2 cost of low-intensity exercise and enhances tolerance to high-intensity exercise in humans. J Appl Physiol 107: 1144-55.

2. Ribeiro F, Alves AJ, Duarte JA, Oliveira J (2010) Is exercise training an effective therapy targeting endothelial dysfunction and vascular wall inflammation? Int J Cardiol 141: 214-21.

3. Lanza GA, Cianflone D, Rebuzzi AG, Angeloni G, Sestito A, et al. (2006) Prognostic value of ventricular arrhythmias and heart rate variability in patients with unstable angina. Heart 92: 1055-63.

4. Huikuri HV, Perkiomaki JS, Maestri R, Pinna GD (2009) Clinical impact of evaluation of cardiovascular control by novel methods of heart rate dynamics. Philos Transact A Math Phys Eng Sci 367: 1223-38.

5. Peng CK, Havlin S, Stanley HE, Goldberger AL (1995) Quantification of scaling exponents and crossover phenomena in nonstationary heartbeat time series. Chaos 5: 82-7.

6. Pincus SM (1991) Approximate entropy as a measure of system complexity. Proc Natl Acad Sci U S A 88: 2297-301.

7. Richman JS , Moorman JR (2000) Physiological time-series analysis using approximate entropy and sample entropy. Am J Physiol Heart Circ Physiol 278: H203949 .

8. Makikallio TH, Tapanainen JM, Tulppo MP, Huikuri HV (2002) Clinical applicability of heart rate variability analysis by methods based on nonlinear dynamics. Card Electro physiol Rev 6: 250-5.

9. Huikuri HV, Jokinen V, Syvanne M, Nieminen MS, Airaksinen KE, et al. (1999) Heart rate variability and progression of coronary atherosclerosis. Arterioscler Thromb Vasc Biol 19: 1979-85.

10. Lampert R, Bremner JD, Su S, Miller A, Lee F, et al. (2008) Decreased heart rate variability is associated with higher levels of inflammation in middle-aged men. Am Heart J 156: 759 e1-7.

11. Frenneaux MP (2004) Autonomic changes in patients with heart failure and in post-myocardial infarction patients. Heart 90: 1248-55.

12. Krstacic G, Krstacic A, Smalcelj A, Milicic D, Jembrek-Gostovic M (2007) The "Chaos Theory" and nonlinear dynamics in heart rate variability analysis: does it work in short-time series in patients with coronary heart disease? Ann Noninvasive Electrocardiol 12: 130-6.

13. Shin DG, Yoo CS, Yi SH, Bae JH, Kim YJ, et al. (2006) Prediction of paroxysmal atrial fibrillation using nonlinear analysis of the R-R interval dynamics before the spontaneous onset of atrial fibrillation. Circ J 70: 94-9.

14. Malik M (1996) Task Force of the European Society of Cardiology and the North American Society of Pacing and Electrophysiology. Heart rate variability: standards of measurement, physiological interpretation and clinical use. Circulation 93: 1043-65.

15. Baumert M, Lambert GW, Dawood T, Lambert EA, Esler MD, et al. (2009) Short-Term Heart Rate Variability and Cardiac Norepinephrine Spillover in Patients with Depression and Panic Disorder. Am J Physiol Heart Circ Physiol 297: H674-9

16. Mourot L, Bouhaddi M, Gandelin E, Cappelle S, Nguyen NU, et al. (2007) Conditions of autonomic reciprocal interplay versus autonomic co-activation: Effects on non-linear heart rate dynamics. Auton Neurosci 137: 27-36.

17. Tulppo MP, Hughson RL, Makikallio TH, Airaksinen KE, Seppanen T, et al. (2001) Effects of exercise and passive head-up tilt on fractal and complexity properties of heart rate dynamics. Am J Physiol Heart Circ Physiol 280: H1081-7. 
18. Pena MA, Echeverria JC, Garcia MT, Gonzalez-Camarena R (2009) Applying fractal analysis to short sets of heart rate variability data. Med Biol Eng Comput 47: 709-17.

19. Goldberger AL, Amaral LAN, Glass L, Hausdorff JM, Ivanov PC, et al. (2000) PhysioBank, PhysioToolkit, and PhysioNet: components of a new research resource for complex physiologic signals. Circulation 101: e215-20.

20. Tulppo MP, Kiviniemi AM, Hautala AJ, Kallio M, Seppanen T, et al. (2005) Physiological background of the loss of fractal heart rate dynamics. Circulation 112: 314-9.

21. Grassi G, Esler M (1999) How to assess sympathetic activity in humans. J Hypertens 17: 719-34.

22. Musialik-Lydka A, Sredniawa B, Pasyk S (2003) Heart rate variability in heart failure. Kardiol Pol 58: 10-3.

23. Braun C, Kowallik P, Freking A, Hadeler D, Kniffki KD, et al. (1998) Demonstration of nonlinear components in heart rate variability of healthy persons. Am J Physiol 275: H1577-84

24. Woo MA, Stevenson WG, Moser DK, Middlekauff HR (1994) Complex heart rate variability and serum norepinephrine levels in patients with advanced heart failure. J Am Coll Cardiol 23: 565-9.

25. Tulppo MP, Makikallio TH, Takala TE, Seppanen T, Huikuri HV (1996) Quantitative beat-to-beat analysis of heart rate dynamics during exercise. Am J Physiol Heart Circ Physiol 271: H244-52.

26. Beckers F, Verheyden B, Aubert AE (2006) Aging and nonlinear heart rate control in a healthy population. Am J Physiol Heart Circ Physiol 290: H2560-70.

27. Porta A, Gnecchi-Ruscone T, Tobaldini E, Guzzetti S, Furlan R, et al. (2007) Progressive decrease of heart period variability entropy-based complexity during graded head-up tilt. J Appl Physiol 103: 1143-9

28. VanTeeffelen JW, Constantinescu AA, Brands J, Spaan JA, Vink H (2008) Bradykinin- and sodium nitroprusside-induced increases in capillary tube haematocrit in mouse cremaster muscle are associated with impaired glycocalyx barrier properties. J Physiol 586: 3207-18.

29. Francis DP, Willson K, Georgiadou P, Wensel R, Davies LC, et al. (2002) Physiological basis of fractal complexity properties of heart rate variability in man. J Physiol 542: 619-29.

30. Makikallio TH, Ristimae T, Airaksinen KE, Peng CK, Goldberger AL, et al. (1998) Heart rate dynamics in patients with stable angina pectoris and utility of fractal and complexity measures. Am J Cardiol 81: 27-31.

31. Mourot L, Bouhaddi M, Regnard J (2009) Effects of the cold pressor test on cardiac autonomic control in normal subjects. Physiol Res 58: 83-91.

32. Tulppo MP, Makikallio TH, Seppanen T, Shoemaker K, Tutungi E, et al. (2001) Effects of pharmacological adrenergic and vagal modulation on fractal heart rate dynamics. Clin Physiol 21: 515-23.

33. Penttila J, Helminen A, Jartti T, Kuusela T, Huikuri HV, et al. (2003) Effect of cardiac vagal outflow on complexity and fractal correlation properties of heart rate dynamics. Auton Autacoid Pharmacol 23: 173-9.

34. Lin LY, Lin JL, Du CC, Lai LP, Tseng YZ, et al. (2001) Reversal of deteriorated fractal behavior of heart rate variability by beta-blocker therapy in patients with advanced congestive heart failure. J Cardiovasc Electrophysiol 12: 26-32.

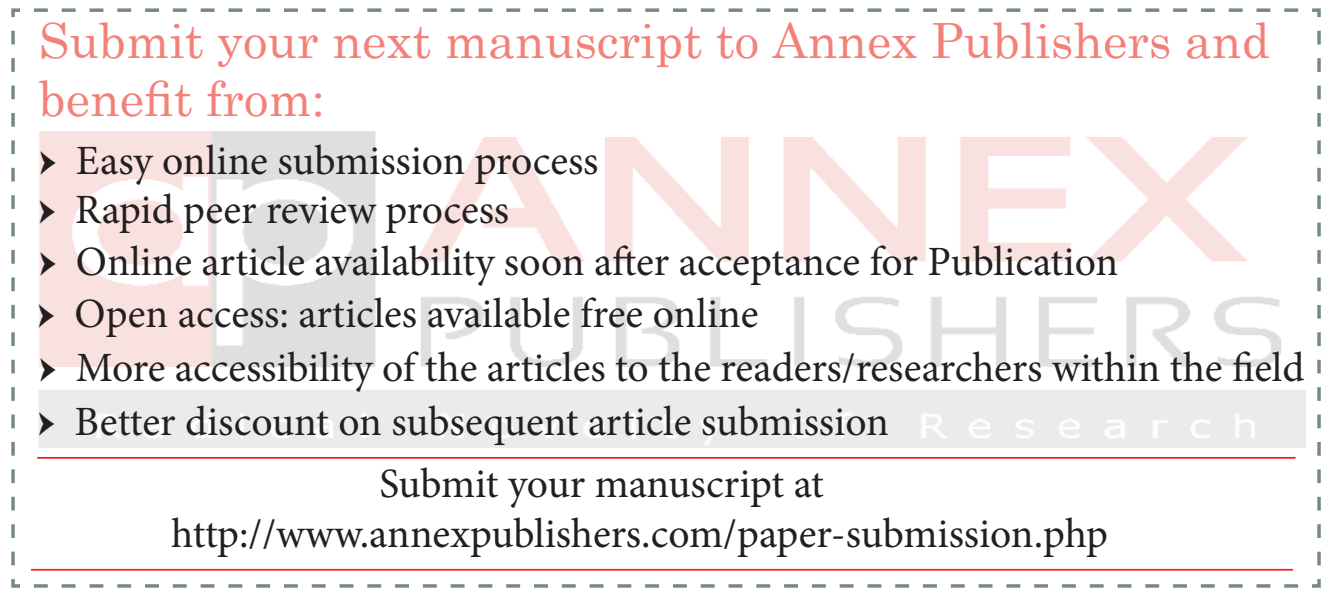

